\title{
Novel protocol for reversing prediabetes and diabetes without medications:The 3-hour post-meal glucose testing
}

\begin{abstract}
Introduction: Type 2 diabetes mellitus (diabetes) is usually caused by insulin resistance and it is considered an incurable life-long disease.

Patient concerns: Diabetes has become a worldwide plague; its prevalence continues increasing without end in sight. Diabetes is the leading cause of blindness, renal failure, lower extremity amputations, peripheral neuropathy, and so on. It is also a major contributor to coronary artery disease, strokes, erectile dysfunction, and premature deaths. Expenses related to diabetic care and patient suffering are staggering. In most cases, patients need to be on medications for life.
\end{abstract}

Diagnosis: Diagnosis and follow-up of diabetes is usually done with A1c hemoglobin.

Interventions: A 3-hour post-meal glucose testing protocol, not previously reported, is described to control and reverse type 2 diabetes. A 3-4year observation of one individual who used this novel protocol is included.

Outcomes: Using the 3-hour post-meal glucose testing protocol, a 58-year-old male reversed early prediabetes $(\mathrm{A} 1 \mathrm{c}=5.7)$ and has been able to maintain a normal A1c of 4.7 on a non-diabetic diet without any caloric restrictions 3-4 years later.

Conclusion: A 3-hour post-meal glucose testing is proposed for prediabetic or diabetic patients to find what fats or meals are blocking their insulin receptors, so that the offending meals and fats can be avoided, and insulin resistance can be reversed. This approach could revolutionize diabetic patient care by empowering patients with a simple and easy to follow protocol.

Keywords: Type 2 diabetes, reversing diabetes, reversing prediabetes, 3-hour glucose protocol
Volume I4 Issue I - 202 I

Manuel Moran

Department of Surgery, Veterans Administration Medical Center, USA

Correspondence: Manuel Moran, 480 IVeterans Drive, Veterans Administration Medical Center, USA, Saint Cloud, MN 56303, USA, Fax I-320-255-6406, Tel I-320-255-6429, Email manuel.moran@va.gov

Received: November 25, 2020 | Published: January 05, 2021
Abbreviations: HOMA-IR, homeostatic model assessment of insulin resistance; diabetes, type 2 diabetes mellitus; A1c, A1c hemoglobin; BMI, body mass index; HIIT, high intensity interval training; MRI, magnetic resonance imaging

\section{Introduction}

Insulin resistance in type 2 diabetes mellitus is caused by fat, as proven by a large body of scientific evidence. Routine screening with A1c hemoglobin, although not routinely done in clinical practice, should allow early detection of prediabetes. An even earlier diagnosis could be established with an insulin resistance test (HOMA-IR $=$ Homeostatic Model Assessment of Insulin Resistance). Early insulin resistance would be the ideal stage to implement preventative measures. The protocol described herein will allow reversing prediabetes and diabetes, normalizing A1c levels. Because no actual cure for the insulin receptor is available, the offending fats that trigger insulin resistance must be avoided. In some patients, though, mineral deficiencies - like chromium - should be corrected as this can eliminate insulin resistance. Multi-ingredient supplements are commercially available (e.g., Nutra-Support Diabetes from Carlson). Many supplements have been found to somewhat improve insulin sensitivity, but their benefit is limited and beyond the scope of this article. Supplements will not reverse insulin resistance if the offending fats are not eliminated.

It was already known early in the $20^{\text {th }}$ century that fats-not carbohydrates - caused insulin resistance. This explains how some modern studies that kept patients on a high carbohydrate diets were able to obtain good glucose control. The first scientific evidence explaining the insulin resistance mechanism was published in the New England Journal of Medicine in 2004. The study showed intramyocellular lipid content in skeletal muscles was blocking the insulin receptor. ${ }^{1}$

In the scientific literature, the main culprit causing insulin resistance is saturated animal fat. ${ }^{2-36}$ Nevertheless; other unhealthy fats have also been found to be contributors.

MRI technology allows the visualization of how fat inside muscle cells blocks the insulin receptor. Intravenous fat injections cause insulin resistance. Decreasing the amount of circulating intravascular fat restores normal insulin receptor function.

Although many people still believe sugar or carbohydrates cause type 2 diabetes, an elevated glucose is only a sign of insulin resistance. Decrease carbohydrate intake is recommended for type 2 diabetic patients because chronically elevated blood glucose levels 
have devastating long term complications but glucose itself does not cause insulin resistance. The role of large amounts of fructose as an etiological factor causing insulin resistance in general, and specifically in the liver, is beyond the scope of this article but it is due to increased lipid levels.

In addition, even healthy fats can block the insulin receptor when heated to certain temperatures. Animal fats cooked at high temperatures further increase type 2 diabetes risks. A study showed that those participants who ate meat and poultry cooked at high temperatures (like grilled, barbecued, etc.) develop more diabetes then those cooking their meals at lower temperatures, despite eating the same amounts of animal products. ${ }^{37}$ Unfortunately, modern medicine focuses on treatment not prevention. Hopefully this article will help reverse this modern trend. The future of Medicine is prevention, not treatment.

\section{Material and methods}

The protocol described herein is based on how long it takes a fatty meal to cause insulin resistance. It takes between 2.5 hours and 3 hours after a fatty meal for the insulin receptor to become less sensitive. Thus, a 3-hour post-meal blood glucose measurement is required to figure out what fats or fatty meals should be avoided. An unhealthy fatty meal can block the insulin for a variable amount of time, from a few hours to several days.

Ideally, patients should improve their blood glucose control as much as possible. Once stable (not necessarily normal) blood glucose is achieved, different fatty meals will be consumed, and 2-hour and 3-hour post-meal blood glucose will be obtained. The 3-hour blood glucose should be lower or not very different than the 2-hour glucose, but not significantly higher. If the 3-hour post-meal glucose is significantly higher (e.g., $>5 \%-10 \%$ ), hourly blood glucose levels will be obtained (e.g., four-hour glucose, etc.) until the blood glucose is less than the previous one. Longer times for the blood glucose to start normalizing mean the fatty meal disrupted more the insulin receptor. In some cases, it might take a few days before a normal blood glucose is obtained. If this happens, new fats are not tested until glucose levels improve. Fats or meals causing 3-hour insulin resistance must be avoided. I have developed this method over several years doing hundreds or thousands of blood glucose tests on myself. This protocol can be used in all patients with prediabetes. It will work in diabetic patients who have insulin resistance but still have high serum insulin levels. In late type 2 diabetes, chronic inflammation can destroy pancreatic beta cells leading to low serum insulin levels. These patients might show some improvement in their insulin resistance (e.g., need for less insulin) but probably will not normalize their hyperglycemia.

\section{Detailed 3-hour protocol}

First, fasting blood glucose should be decreased as much as possible. The best way is to do a 5-day fast. It can be water fast (with broth for adequate salt consumption) or less than 400 calories/day, eating mostly vegetables in general and specifically many green leafy vegetables. Healthy vegetables include celery, cabbage, kale, spinach, broccoli, and sauerkraut, but many other are acceptable. This modified fasting option, less than 400 calorie/day, is much easier for patients to follow than water fasting. In either case, protein will be avoided or minimize to maximize autophagia.

A fat-free salad dressing is useful to decrease total daily caloric intake. For example, mixing half a cup of organic coconut aminos with the same amount of organic apple cider vinegar. Then, adding liquid stevia (may use monk fruit instead) for better taste. May add a crushed garlic clove. The salad dressing is ready for use. A more concentrated dressing will be obtained by simmering the solution for a few minutes until half the volume has evaporated. In this case, stevia should be added after simmering.

A 5-day fast will expedite achieving a normal blood glucose levels and has many other health benefits, like stimulating the release of stem cells. Patients with significant chronic medical conditions could do a 5-day fast up to once a month. After the 5-day fast, the patient should start an organic whole-food plant-based diet, low in fat ( $10 \%$ of calories). Initially, no fats or oils should be heated. It is very important to avoid all processed foods. Patients must void all unhealthy fats, like processed vegetable oils (canola, corn, cottonseed, safflower, sunflower, soybean), peanut oil, all man-made oils and fats (hydrogenated oils, trans fats, interesterified fats, Crisco), and healthy fats that have been heated. Extra virgin olive oil and organic cold press coconut oil are acceptable but should be minimized, at least in the beginning. Vegetables may be safely steamed. Ideally, food should be raw the first month, although steaming vegetables is acceptable and recommended for certain vegetables (kale, spinach, cauliflower, broccoli, etc.)

An alternative option, although maybe not as ideal, is to do a 5-day mimicking diet, as described by Dr. Valter Longo. In this case, about 800 calories of a plant-based diet are consumed daily. This fast mimicking diet is also commercially available (Prolon at https:// prolonfmd.com/).

If a patient is not willing to do a 5-day fast, then start an organic whole-food plant-based diet, low in fat (10\% of calories). Again, initially fats and oils should not be heated. Once a stable glucose is achieved, low temperature cooking can be tested to see how it affects the insulin receptor. High temperature cooking is rather unhealthy and should always be avoided. Unhealthy fats (like partially hydrogenated oils, processed vegetable oils, etc.) should not be ingested, not even after blood glucose levels and A1c hemoglobin have normalized. Healthy heat-sensitive fats, like omega 3 based fats, should not be heated at all. This diet will be low calorie in the beginning, which also helps normalizing blood glucose levels, but there is no need to count calories. Total caloric intake can be increased slowly until a patient's specific caloric and/or fat threshold have been reached. Continuous glucose monitoring devices will facilitate this protocol but are not mandatory. Unfortunately, insurance companies will not cover these devices unless a patient already has been diagnosed with diabetes.

\section{Results}

58-year-old male with no history of diabetes or prediabetes was found to have an A1c of 5.7. Prediabetes is considered A1c of 5.7 to 6.4 No other medical conditions. Normal BMI, on no prescription medications. Normal studies included lipid panel, homocysteine, uric acid, fibrinogen, and highly sensitive $\mathrm{C}$ reactive protein. No family history of diabetes mellitus.

Patient was started on this protocol, including fasting, as described above. Later, diet remained mostly plant-based diet (about 10\% of calories from animal products) without any restrictions on caloric intake or percentage of fat ingested. A1c remains at 4.7 after more than three and a half years after intervention started. A ketogenic diet was used for about one year (total net carbohydrates $=50-60$ grams/ day) but at this time the diet followed does not have any non-refined carbohydrates restrictions. Patient avoids all refined carbohydrates, 
like sugar, flowers, bread, pasta, etc., but this is done for health reasons, not to improve insulin sensitivity.

\section{Fats that caused insulin resistance}

The most significant insulin resistance was caused by sweet and sour Chinese food (chicken) purchased at a take-out restaurant, with a 2-hour blood glucose of 161 and a 3-hour blood glucose $>200$. No desert or refined carbohydrates were ingested with this Chinese meal. After this meal, it took 3-4days for fasting blood glucose levels to normalize. These high glucose levels can be explained by the type of oil used to cook this meal (processed vegetable oil that frequently restaurants reuse many times, thus oxidizing the oil).

Other meals or fats that caused insulin resistance included four soft boiled eggs, two tablespoons of commercially processed almond butter (raw, not roasted), homemade milk chocolate heated during grinding to 140 degrees Fahrenheit for a 24-hour period, and healthy fats heated to high temperatures. Interestingly, dark chocolate from ChocZero (Keto Barks) also caused insulin resistance (2-hour glucose around 120, but $>140$ at three hours) on several occasions despite this chocolate not having any sugar or animal fats. This would be expected if fats used for the chocolate were significantly heated during the manufacturing process. Minor insulin resistance was detected after ingesting $30 \mathrm{ml}$ of high-quality non-heated fish oil, indicating that even healthy oils in large amounts can affect the insulin receptor. Many hundreds of tests failed to reveal any significant insulin resistance in the first two hours after a fatty meal. Post-meal testing at 2.5 hours was not performed.

\section{Fats that did not cause insulin resistance}

No insulin resistance was detected after eating 4-6 egg yolks eaten raw (boiled did cause insulin resistance as mentioned above), homemade milk chocolate ground for 24 hours at temperatures $<100$ degrees (cocoa nibs were not roasted), organic coconut oil $(3 \mathrm{ml})$, macadamia oil $(3 \mathrm{ml})$, extra virgin olive oil $(30 \mathrm{ml})$, flax seed oil (30 ml), $5-10 \mathrm{ml}$ of fish oil, organic butter ( $30 \mathrm{~g}$, not heated), small portions of animal products cooked a low temperatures (veal, bison, chicken), mayonnaise (made with avocado oil, no other oils used as ingredients), and various non-roasted nuts (pecans, almonds, brazil nuts, hazelnuts, macadamia, and walnuts.)

\section{Discussion}

Type 2 diabetes is not curable with current technology, but it should be possible for patients to find and avoid those fats that are giving them insulin resistance. The many diabetic long-term complications including neuropathy, blindness, kidney failure, amputations, myocardial infarctions, strokes, erectile dysfunction, etc., should be a good incentive for prediabetic patients to follow simple medical and nutritional recommendations. Without appropriate life-style modifications, many patients with prediabetes will end up with full blown diabetes. It is estimated that $70 \%$ of patients with prediabetes will have full-blown diabetes ten years later.

I propose that reversing prediabetes is possible by using a 3-hour post-meal insulin testing protocol. Of course, well design programs to maintain or achieve an ideal body weight and to exercise should be implemented. HIIT (high intensity interval training) is ideal. Unhealthy food options, like unhealthy fats and processed foods, should be avoided. Cooking should be done at low temperatures as proven by the same fat causing insulin resistance only after it had been heated (egg yolks, chocolate). Fast food restaurants should be avoided. Food cooked in a healthy way and with healthy ingredients in a good restaurant is probably acceptable, but patients should confirm it by doing the 3-hour test. The role of heavy metal and persistent organic pollutants detoxification is beyond the scope of this article but should be considered.

Screening A1C should be widely implemented to allow reversal of pre-diabetes in the early stages when is much easier than when somebody already has more advanced disease. Health insurance companies should cover A1C screening and the proposed 3-hour postmeal glucose testing. Even better, an insulin resistance test (HOMAIR) will allow counseling at earlier stages of insulin resistance. Randomized trial will need to be done.

\section{Conclusion}

A 3-hour post-meal glucose testing is proposed for prediabetic or diabetic patients to determine what fats or meals are blocking their insulin receptors. This method will allow patients to avoid offending fats and meals so that insulin resistance can be reversed. This approach could revolutionize diabetic patient care. Randomized clinical trials are needed to validate the effectiveness of the proposed 3-hour postmeal glucose testing protocol.

\section{Acknowledgments}

None.

\section{Conflicts of interest}

None. Author has no pertinent financial interests with the subject matter or materials discussed.

\section{Funding}

None.

\section{References}

1. Petersen KF, Dufour S, Befroy D, et al. Impaired mitochondrial activity in the insulin-resistant offspring of patients with type 2 diabetes. $N$ Engl J Med. 2004;350(7):664-671.

2. Sweeney S. Dietary factors that influence the dextrose tolerance test. A preliminary study. Arch Intern Med. 1927;40(6):818-830.

3. Krssak M, Petersen FK, A Dresner A, et al. Intramyocellular lipid concentrations are correlated with insulin sensitivity in humans: a $1 \mathrm{H}$ NMR spectroscopy study. Diabetologia. 1999;42(1):113-116.

4. Roden M, Price TB, Perseghin G, et al. Mechanism of Free Fatty Acidinduced insulin Resistance in Humans. J Clin Invest. 1996;97(12):28592865.

5. Roden M, Krssak M, Stingl H, et al. Rapid Impairment of Skeletal Muscle Glucose Transport/Phosphorylation by Free Fatty Acids in Humans. Diabetes.1999;48(2):358-364.

6. Lee S, Boesch C, Kuk JL, et al. Effects of an overnight lipid infusion on intramyocellular lipid content and insulin sensitivity in Africn-American versus Caucasian adolescents. Metabolism. 2013;62(3):417-423.

7. Santomauro AT, G Boden, M E Silva, et al. Overnight Lowering of Free Fatty Acids With Acipimox Improves Insulin Resistance and Glucose Tolerance in Obese Diabetic and Nondiabetic Subjects. Diabetes. 1999;48(9):1836-1841.

8. Roden M. How Free Fatty Acids Inhibit Glucose Utilization in Human Skeletal Muscle. News Physiol Sci. 2004;19:92-96.

9. Bachmann OP, Dahl DB, Brechtel K, et al. Effects of Intravenous and Dietary Lipid Challenge on Intramyocellular Lipid Content and the Relation With Insulin Sensitivity in Humans. Diabetes. 2001;50(11):25792584. 
10. Samuel VT, Shulman GI. Mechanisms for Insulin Resistance: Common Threads and Missing Links. Cell. 2012;148(5):852-871.

11. Rachek LI. Free Fatty Acids and Skeletal Muscle Insulin Resistance. Prog Mol Biol Transl Sci. 014;121:267-292.

12. vans WJ. Oxygen-Carrying Proteins in Meat and Risk of Diabetes Mellitus. JAMA Intern Med. 2013;173(14):1335-1336.

13. Perseghin G, Scifo P, Cobelli FD, et al. Intramyocellular Triglyceride Content Is a Determinant of in Vivo Insulin Resistance in Humans. Diabetes. 1999;48(8):1600-1606.

14. Nolan CJ, Larter CZ. Lipotoxicity: Why do saturated fatty acids cause and monounsaturated protect against it? $J$ Gastroenterol Hepatol. 2009;24(5):703-711.

15. Ye J. Role of Insulin in the Pathogenesis of Free Fatty Acid-Induced Insulin Resistance in Skeletal Muscle. Endocr Metab Immune Disord. 2007;7(1):65-74

16. Estadella D, Oller do Nascimento CMP, Oyama LM, et al. Lipotoxicity: Effects of Dietary Saturated and Transfatty Acids. Mediators Inflamm. 2013;2013:137579

17. Vessby B, Uusitupa M, Hermansen K, et al. Substituting dietary saturated for monounsaturated fat impairs insulin sensitivity in healthy men and women: The KANWU study. Diabetologia. 2001;44(30:312-319.

18. Martins AR, Nachbar RT, Gorjao R, et al. Mechanisms underlying skeletal muscle insulin resistance induced by fatty acids:importance of the mitochondrial function. Lipids Health Dis. 2012;11:30.

19. Karlic H, Schuster D, Varga F, et al. Vegetarian Diet Affects Genes of Oxidative Metabolism and Collagen Synthesis. Ann Nutr Metabol. 2008;53:29-32.

20. Goff LM, Bell JD, So PW, et al. Veganism and its relationship with insulin resistance and intramyocellular lipid. Eur J Clin Nutr. 2005;59(2):291298.

21. Gojda J, Patková J, Jaček M, et al. Higher insulin sensitivity in vegans is not associated with higher mitochondrial density. Eur J Clin Nutr. 2013;67(12)1310-1315.

22. West KM, Kalbfleisch JM, et al. Influence of Nutritional Factors on Prevalence of Diabetes. Diabetes.1971;20(2):99-108.

23. Snowdon DA, Phillips RL. Does a Vegetarian Diet Reduce the Occurrence of Diabetes? Am J Public Health. 1985;75(5):507-512.

24. Tonstad S, Stewart K, Oda K, et al. Vegetarian diets and incidence of diabetes in the Adventist Health Study-2. Nutr Metab Cardiovasc Dis. 2013;23(40:292-299.
25. Chiu THT, Huang HY, Chiu YF, et al. Taiwanese Vegetarians and Omnivores:Dietary Composition, Prevalence of Diabetes and IFG. PLoS One. 2014;9(2):e88547.

26. Cunha DA, Igoillo-Esteve M, Gurzov EN, et al. Death Protein 5 and p53Upregulated Modulator of Apoptosis Mediate the Endoplasmic Reticulum Stress-Mitochondrial Dialog Triggering Lipotoxic Rodent and Human beta cell Apoptosis. Diabetes. 2012;61(11):2763-2775.

27. Xiao C, Giacca A, Carpentier A, et al. Differential effects of monounsaturated, polyunsaturated and saturated fat ingestion on glucosestimulated insulin secretion, sensitivity and clearance in overweight and obese, non-diabetic humans. Diabetologia. 2006;49(6):1371-1379.

28. Evans WJ. Oxygen-Carrying Proteins in Meat and Risk of Diabetes Mellitus. JAMA Intern Med. 2013;173 (14):1335-1336.

29. Cao J, Feng XX, Yang NB, et al. Saturated Free Fatty Acid Sodium Palmitate-Induced Lipoapoptosis by Targeting Glycogen Synthase Kinase-3B Activation in Human Liver Cells. Dig Dis Sci. 2014;59(2):34657.

30. Parker DR, Weiss ST, Troisi R, et al. Relationship of dietary saturated fatty acids and body habitus to serum insulin concentrations:the Normative Aging Study. Am J Clin Nutr. 1993;58(2):129-136.

31. Maron DJ. Saturated Fat Intake and Insulin Resistance in Men With Coronary Artery Disease. Circulation. 1991;84(5):2020-2027.

32. Wang L, Folsom AR, Zheng ZJ, et al. Plasma fatty acid composition and incidence of diabetes in middle-aged adults:the Atherosclerosis Risk in Communities (ARIC) Study. Am J Clin Nutr. 2003;78(1):91-98.

33. Taylor R. Pathogenesis of type 2 diabetes:tracing the reverse route from cure to cause. Diabetologia. 2008;51(10):1781-1789.

34. Feskens EJ, Sluik D, Woudenbergh GJ. Meat Consumption, Diabetes, and Its Complications. Curr Diab Rep. 2013;13(2):298-306.

35. Palli BB, Masala G, Sharp SJ, et al. Association between dietary meat consumption and incident type 2 diabetes:the EPIC-InterAct study. The InterAct Consortium. Diabetologia. 2013;56(1):47-59.

36. Zoncu R, Efeyan A, Sabatini DM, et al. mTor: from growth signal integration to cancer, diabetes and ageing. Nat Rev Mol Cell Biol. 2011;12:21-35.

37. Liu G, Zong G, Wu K, et al. Meat Cooking Methods and Risk of Type 2 Diabetes: Results from Three Prospective Cohort Studies. Diabetes Care 2018;41(5):1049-1060 